\title{
Towards Securing Availability in 5G: Analyzing the Injection Attack Impact on Core Network
}

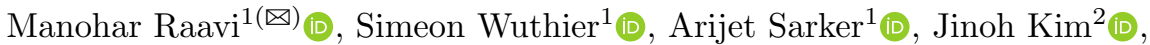 \\ Jong-Hyun $\mathrm{Kim}^{3}{ }^{\mathbb{D}}$, and Sang-Yoon Chang ${ }^{1}$ (1) \\ 1 University of Colorado, Colorado Springs, CO 80918, USA \\ \{mraavi, swuthier, asarker, schang2\}@uccs.edu \\ 2 Texas A \& M University, Commerce, TX 75428, USA \\ jinoh.kim@tamuc.edu \\ ${ }^{3}$ Electronics and Telecommunications Research Institute, Daejeon, South Korea \\ jhk@etri.re.kr
}

\begin{abstract}
G technology for mobile networking involves control communications to set up the radio channels and the authentication and security credentials. The control communications preceding the authentication and subscription verification remain vulnerable against the communication injection threats. We study the injection threats on control communications in 5G New Radio standard in 3GPP. From our 5G clientbased implementation and experimentation against real-world networking, we analyze and measure the threat impact against the $5 \mathrm{G}$ service provider infrastructure of the core network. To secure 5G networking, our paper discusses about future research directions for increasing the understanding of such vulnerability/threat and for building greater security and availability for $5 \mathrm{G}$ networking against such wireless injection threats.
\end{abstract}

Keywords: $5 \mathrm{G} \cdot$ Mobile networking $\cdot$ Communication $\cdot$ Injection attack $\cdot$ DoS

\section{Introduction}

Mobile users use cellular networking to access the Internet and network with other computers. Since the 2G supporting text messaging in the early 1990's, cellular technologies have been evolving with the subsequent generation of technologies with increasing performances in communication rate, scalability/density in the number of users, and broader set of applications. This paper focuses on the most recent cellular technology in $5 \mathrm{G}$ networking.

In computer networking, the user devices use the control communications to set up the communication channels before delivering the goodput data using the channels. The control communications include the synchronization and the agreement in both the channel resources (such as the frequency band/bandwidth

(C) The Author(s) 2022

S.-Y. Chang et al. (Eds.): SVCC 2021, CCIS 1536, pp. 143-154, 2022.

https://doi.org/10.1007/978-3-030-96057-5_10 


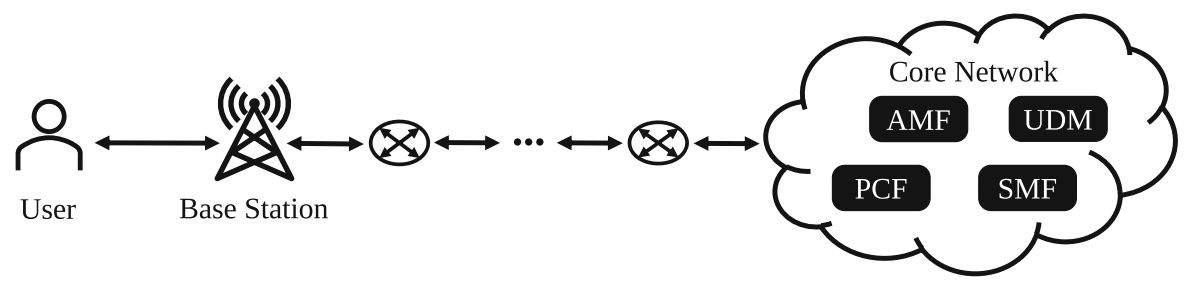

Fig. 1. The networking illustration including user, base station, and the core network. The communications between the user and base station are wireless (the furthest left arrow in the diagram), while the rest of the communication links are wired (the other arrows). Our injection threat affects all the communication links between the user and the core network.

and the modulation type), the security setup and credentials (such as the security mechanisms/protocol type and the key), and the application-layer registration. As shown in Fig. 1, in 5G and earlier cellular technologies, the control communications begin with the user initiating the channel request and the wireless channel setup, which involves the user and base station. Afterward, the control communication involves the core network beyond the base station for registration processing/verification and the security setup/key exchange. Therefore, the earlier the control communications before the security setup and authentication and the corresponding protection remain vulnerable against injection attacks.

In this paper, we study the security threat based on injections in the $5 \mathrm{G}$ control communications against the 5G networking service provider's availability. We show that the injection attack is feasible in the current 5G networking and quantify how much resources it consumes against the networking availability. While multiple injections lead to flooding for DoS attack, we focus on per injection and the corresponding resource consumptions at both the attacker (client) and the victim (the service provider end). To analyze and estimate the injection threat impact, we experiment on the real-world 5G networking to provide reference measurements and estimate the injection attack impact based on those measurement results.

The rest of the paper is organized as follows. Section 2 provides the background information on $5 \mathrm{G}$ control communications and Sect. 3 describes the injection threat against 5G. Section 4 provides the experiment methodology used, discusses measurement results, and estimates the injection impact. We review the related work on control communications security in Sect. 5 and discuss the future scope and potential countermeasures in Sect. 6. Finally, we conclude our work in Sect. 7. 


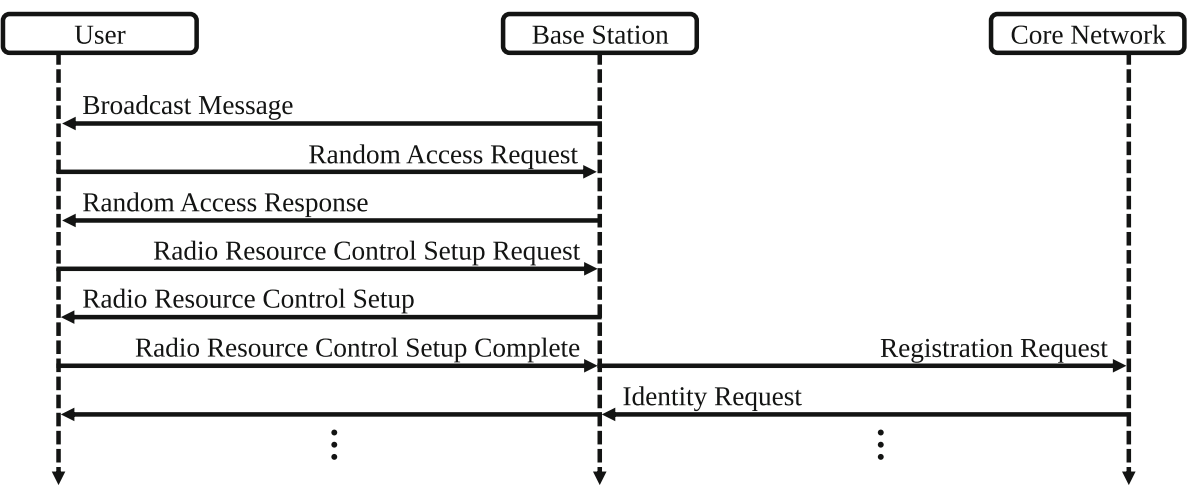

Fig. 2. 5G control communications including the wireless/radio access setup and the user registration verification $[2,4]$. This diagram focuses on the parts of the communication most relevant to our work, which are the parts before establishing the security and key exchange. The control communications further encapsulate the rest of the registration with "...", including authentication and security mode setup. The data communication for the actual $5 \mathrm{G}$ service/goodput follows after control communications.

\section{Background of 5G Control Communications}

In $5 \mathrm{G}$, the user needs to establish control communications with the base station and core network to access the network services [2,4]. The control communications build on the a priori registration, which occurs before the real-time control communications described in Fig. 2; in such a priori registration, the user obtains a subscriber identity module (SIM) and its SIM registration gets inputted to the Core Network system. The real-time control communications which occur when the user wants to start networking for cellular service access verifies the user identity/subscription within the core network.

As shown in Fig. 2, these control communications involve performing channel setup with the base station and sending a registration request to the core network. Each of the individual control messages contains different parameters as listed in Table 1. The user synchronizes the downlink information from the broadcast messages (or synchronization signals) of the base station and starts a random access procedure for uplink connection during channel setup. There are 64 possible preambles defined in [3] and user equipment receives this information from broadcast messages.

For random access procedure initialization, the user sends a random access request to the base station with a preamble (randomly selected from the 64 preambles). In contention-free random access, the base station assigns a preamble. The base station prepares a random access response with allocated identifiers and provides resources to the user for further communications after the reception of the random access request from the user. The random access response is notified to the user through downlink control channel and carried on downlink shared channel. 
Table 1. Information included in the $5 \mathrm{G}$ control communications transactions $[2,4]$.

\begin{tabular}{l|l}
\hline Message & Information includes \\
\hline Broadcast message & $\begin{array}{l}\text { Cell ID, master information block, system } \\
\text { information block }\end{array}$ \\
\hline Random access request & Random access preamble ID \\
\hline Random access response & $\begin{array}{l}\text { Frequency and time resource assignment, } \\
\text { Cell-Radio Network Temporary Identifier } \\
\text { (CRNTI) }\end{array}$ \\
\hline Radio resource control setup request & User identity, Establishment reason \\
\hline Radio resource control setup & $\begin{array}{l}\text { Radio bearers configuration, master cell } \\
\text { information }\end{array}$ \\
\hline Radio resource control setup complete & Acknowledgement \\
\hline Registration request & $\begin{array}{l}\text { Registration type, 5G-globally unique } \\
\text { temporary identifier, user capabilities }\end{array}$ \\
\hline Identity request & $\begin{array}{l}\text { Identity request message identity, identity } \\
\text { type }\end{array}$ \\
\hline
\end{tabular}

The user, on receiving the random access response, sends a radio resource control connection setup request to the base station with a random number (ranges from 1 to $2^{39}-1$ ) for the user identity (called User-Equipment Identity in the 3GPP standard $[2,4]$ ) and establishment reason. The response (radio resource control setup) from the base station contains radio bearers configuration and master cell information. Once the user receives the information, it configures the connection and sends a radio resource control connection complete message along with a registration request to the base station.

The base station forwards the registration request of the user to the core network. The core network has different logical functions to perform identity-check, authentication of the user, security setup, and user capability inquiry before accepting the registration. On successful registration, core network schedules the resources required to provide service to the user. Few of the core network functions are listed in Table 2 with the terminology used in our paper and their equivalents in 3rd Generation Partnership Project (3GPP) standards.

\section{Injection Threat Against 5G}

In this section, we describe and explain the threat model, the injection threat mechanism, and the vulnerability enabling the injection.

Threat Model. We assume a standard active wireless attacker model in that the attacker has the injection capability for generating and transmitting the wireless packets in $5 \mathrm{G}$ (which requires the radio/antenna frontend hardware and the wireless signal processing to generate wireless signals complying with the $5 \mathrm{G}$ 
Table 2. Terminology used in our work and their equivalents in 3rd Generation Partnership Project (3GPP) standards $[1,2,4]$.

\begin{tabular}{l|l}
\hline Our work & 3GPP standards \\
\hline User & User Equipment (UE) \\
\hline Base station & gnodeB (gNB) \\
\hline Core network & $\begin{array}{l}\text { Access and Mobility Management Function (AMF), } \\
\text { Session Management Function (SMF), Unified Data } \\
\text { Management (UDM), and Policy Control Function (PCF) }\end{array}$ \\
\hline Identity request & Non-Access Stratum (NAS) Identity Request \\
\hline
\end{tabular}

NR standard) and can detect and listen another legitimate user's transmission (passive radio receiving capability). In our threat model, the attacker knows the $5 \mathrm{G}$ protocols including the structure of control communication messages and preambles by Kerckhoff's principle. The attacker is implemented on the user side within the communication range to the base station; thus, there is no need to compromise the service provider infrastructure, e.g., the base station itself or a router between the base station and the core network. The attacker generates and transmits the preamble, initial wireless packets, and registration request to engage the base station and core network for Registration Request (from the base station's Broadcasting Message to the attacker user's Identity Request in Fig. 2). The injection attack triggers computing and networking loads to the $5 \mathrm{G}$ service provider infrastructure including the base station and the core network.

The Vulnerability. The vulnerability of our injection threat is from the initial (wireless) channel access setup forgoing any security protection. This is a part of the registration process and control communications before the authentication and the security mode setup, described in Sect. 2. While the attacker only engages the base station for the injection attack, the attack impacts the entire $5 \mathrm{G}$ networking service provider including the core network.

High-Feasibility Threat. Our threat is a high-risk threat since it involves a very low-barrier attack setup/feasibility as discussed in this section while providing great impact on the networking service provider. Our paper focuses on quantifying and measuring the injection attack impact. We focus on the per-injection attack impact analyses. An attacker model with greater constraints (and thus lower feasibility) and with potentially greater sophistication, e.g., those requiring real-time eavesdropping enabling spoofing, may have greater attack impacts, which analyses and studies we leave for future work.

\section{Threat Impact Measurements and Analyses}

In this section, we analyze the attack impact on the base station and core networking based on our cellular networking experimentation measurements. 


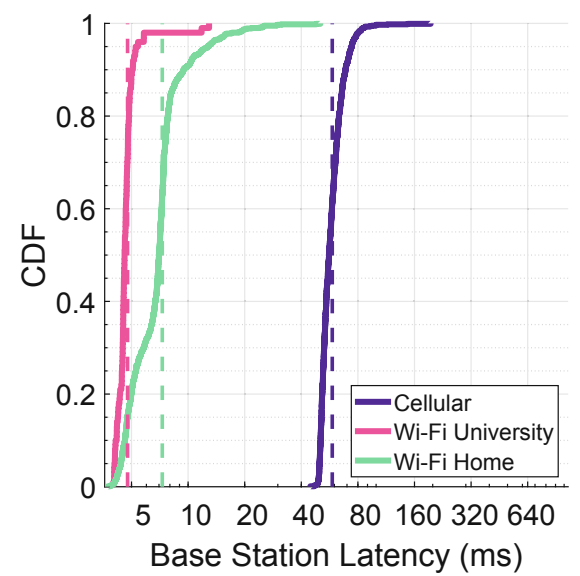

(a) Latency distribution for the base station. The confidence intervals with $95 \%$ certainty for base station are $\pm 0.586 \mathrm{~ms}$, $\pm 0.2234 \mathrm{~ms}$, and $\pm 0.2202 \mathrm{~ms}$ for Cellular, Wi-Fi University, and Wi-Fi Home latencies, respectively.

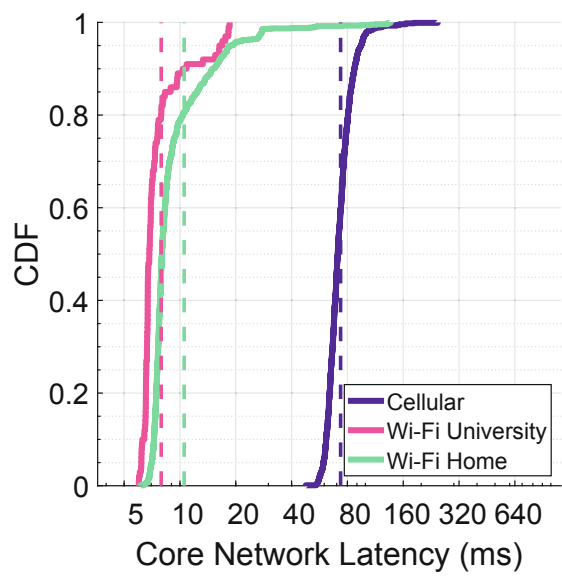

(b) Latency distribution for the core network. The confidence intervals with $95 \%$ certainty for core network are $\pm 0.8486 \mathrm{~ms}, \pm 0.578 \mathrm{~ms}$, and $\pm 0.6669 \mathrm{~ms}$ for Cellular, Wi-Fi University, and Wi-Fi Home latencies, respectively.

Fig. 3. The cumulative distribution function (CDF) for the initial base station/core network node associated with cellular network, university $\mathrm{Wi}-\mathrm{Fi}$, and home $\mathrm{Wi}-\mathrm{Fi}$. The dotted line shows the mean latencies for each platform.

\subsection{Experiment Methodology}

We experiment on real-world networking from the client perspective to estimate the injection attack impact, i.e., we take networking measurements on the client node networking with the $5 \mathrm{G}$ cellular service providers and estimate the attack impact based on the measurements. Because we experiment against real-world networking, we avoid DoS'ing or negatively impacting the service providers and only collect the reference measurements for our injection impact estimation. We select the top ten most used web domains [5] as of August 2021, and run the built-in traceroute shell command to identify every node in the route along with the link latencies. Our measurements are taken using a physical machine with Apple M1 chip, 8 core CPU (4 performance cores and 4 efficiency cores), 16 GBs (LPDDR4) of RAM running Python 3.8 for experimentation automation. While we focus our study on 5G cellular technology ("Cellular"), we do a comparison analyses with $\mathrm{Wi}-\mathrm{Fi}$ and more specifically focusing on the university Wi-Fi networking infrastructure ("Wi-Fi University") and the residence Wi-Fi infrastructure ("Wi-Fi Home") since the university networking infrastructure and the residential networking exhibit different networking behavior and characteristics [11]. The actual service providers are anonymized on this manuscript because we are studying and simulating threats and our threat is applicable to $5 \mathrm{G}$ networking and not service-provider specific. 
We run our experiment for the aforementioned networking cases and collect 1000 samples for each case. We identify the core network nodes from the networking service providers by cross-checking with the service provider IP range from Internet Assigned Numbers Authority [14] and then measure the number of hops and the latencies to reach those core network servers.

\subsection{Networking Measurements for References}

We compare the $5 \mathrm{G}$ networking with Wi-Fi networking, as Wi-Fi has existed longer and is more widely studied in networking. Cellular networking is generally with greater time costs than the Wi-Fi networking (which observation corroborates with [22]) and thus the threat impact is greater than the DoS impact on Wi-Fi. Figure 3a shows the distribution of latencies with regards to the base station. The base station mean latencies for cellular, university Wi-Fi, and home Wi-Fi are 58.45, 4.77, and $7.29 \mathrm{~ms}$, respectively. Our results show that the Wi-Fi-based measurements have the lower latencies (12.25 and 8.02 times faster than cellular for university and home Wi-Fi, respectively), with university being 1.53 times faster than home $\mathrm{Wi}-\mathrm{Fi}$, on average.

Figure $3 \mathrm{~b}$ shows the distribution of latencies with regards to the core network. The core network mean latencies for cellular, university Wi-Fi, and home Wi$\mathrm{Fi}$ are $73.51,7.93$, and $10.54 \mathrm{~ms}$, respectively. We find that the Wi-Fi-based measurements have the lowest latencies (9.27 and 6.97 times faster than cellular for university and home Wi-Fi, respectively) with university being 1.33 times faster than home $\mathrm{Wi}-\mathrm{Fi}$, on average.

Our estimation analysis in Sect. 4.3 focuses on the cellular measurements and we denote the number of communications between the user and base station (one interaction involving back-and-forth communications) as $C_{B S}$ and the number of communications between the base station and core network as $C_{C N}$. We also denote the number of hops between the user and base station as $H_{B S}$ while we use $H_{C N}$ for the number of hops between the user and the core network. Finally, we use $T_{B S}$ to denote the base station latency and $T_{C N}$ to denote the core network latency. From our measurements, we have $H_{B S}=1$ hop (typical since the user directly communicates with the base station via wireless communication link), $H_{C N}=4$ hops, $T_{B S}=58.45 \mathrm{~ms}, T_{C N}=73.51 \mathrm{~ms}$.

\subsection{Injection DoS Impact Estimation}

We estimate the injection attack impact using our networking reference measurements in Sect. 4.2. More specifically, we quantify and estimate the injection attack impact in the number of communications (one back-and-forth communications regardless of whether it is a base station or a cloud-based core network), $C$, the number of hops, $H$, and latency, $T$. We compute $C$ by calculating the total number of round-trip communications to the base station and core network, i.e., $C=C_{B S}+C_{C N}$. For example, from Fig. 2, the number of round-trip communications to the base station, $C_{B S}$, is 2 and to the core network, $C_{C N}$, is 1 , giving $C=3$ (until identity request). If the attacker chooses to send a response, it would 
Table 3. Injection Attack Impact Estimations in the number of communications, $C$, the number of hops $H$, and latency $T$.

\begin{tabular}{l|l|l|l}
\hline Identity response & $\mathrm{C}$ (communications) & $\mathrm{H}$ (hops) & $\mathrm{T}(\mathrm{ms})$ \\
\hline Without & 3 & 12 & 190.41 \\
\hline With & 4 & 20 & 263.92 \\
\hline
\end{tabular}

cost an additional round trip time. Similarly, the total number of hops involved in a round-trip communication is given as twice the product of the number of hops and the number of communications, $H=2 \cdot\left(C_{B S} \cdot H_{B S}+C_{C N} \cdot H_{C N}\right)$. The total latency is given as the product of the number of communications to the base station/core network and their respective latencies, $T=T_{B S} \cdot C_{B S}+$ $T_{C N} \cdot C_{C N}$. Using the above equations, we quantify the injection impact. Table 3 computes the injection impact based on our reference measurements in Sect. 4.2 when an attacker does an injection without and with identity response. If the attacker injects a false identity response, it's going to cause an additional roundtrip communication involving 8 more hops and taking $T=263.92 \mathrm{~ms}$ in total.

\section{Related Work}

In wireless and mobile security, previous research studied the control communication injection threats targeting the victim's availability. These research studies defended against DoS threats based on jamming the control communication channel itself because the control communication channel is publicly known $[6,9,16]$, injecting false information on the wireless-channel-setting medium access control (MAC) communications for DoS [8,19], and injecting false information on the MAC feedback [23]. While our threat can classify as false/bogus control communication injection (more specifically, false requests for the initial channel requests), our work focuses on the emerging $5 \mathrm{G}$ networking as opposed to the more general wireless networking.

In $5 \mathrm{G}$ networking, similar to previous networking technologies, most of the well-known attacks like spoofing, sniffing, signaling, amplification are applicable $[17,18,24]$. The authors of [18] provide threat assessment and mitigation techniques for each of the control channels (broadcast, random access, uplink control, and downlink control) and data channels (uplink and downlink) of 5G. Their assessment shows that spoofing/jamming/sniffing attack efficiency on control channels is more effective. A signaling DoS attack on 3G/WiMax is presented in [17], where a 40-byte packet is sent to 24,000 mobile devices after every $5 \mathrm{~s}$, which generates enough data to overload the used wireless infrastructure. The authors of [24] implement the signaling attack on $4 \mathrm{G}$, classified the impact levels, and discussed possible countermeasures. The authors in [20] show that distributed DoS poses a big threat for $5 \mathrm{G}$ network slices. They also discuss the optimal placement for virtual network functions for guaranteed end-to-end delays. A strategic approach based on game theory is proposed in [21] to secure 
a $5 \mathrm{G}$ control plane from distributed DoS signaling attacks. It involves scaling up or increasing the resource assignment for virtual hosts based on the incoming signaling traffic.

\section{Future Work and Potential Countermeasure Discussions}

We discuss the future scope and potential countermeasures for strengthening the security of control communication. We intend to inform the $5 \mathrm{G}$ standard technologies of the injection threat in order to inform the research and developments for securing the networking availability. To achieve such a goal, we identify the following future research directions:

More Sophisticated Threats. Our threat model is of high feasibility merely requiring the $5 \mathrm{G}$ wireless communication capability, as described in Sect.3. A more sophisticated threat may provide a stronger attack impact on the $5 \mathrm{G}$ service provider's availability but may impose greater constraints for the attacker setup.

Further Injection DoS Impact Analyses. Our work focuses on the perinjection impact analyses due to our experimental setup, including clientnode implementation and experimentation against the real-world networking (Sect.4.1), which can be used to estimate the attacker cost vs. impact for DoS involving multiple injections. Future research can therefore study the threat impact analyses of multiple injections, including flooding, DDoS, and their impacts on the victim's bandwidth and other networking resources. Analyzing the DoS impact on specific parts of the networking infrastructure, e.g., base station and a core network server functionality, can also identify the bottleneck vulnerabilities of the service provider infrastructure. Furthermore, there can be other threat impact metrics, including the wireless channel/mediumaccess-control resources (between the user and the base station) [8,17-19,23], host-based networking resources (similar to a TCP SYN Flood attacking the server connection table) $[7,15,18,25]$, and the power resource (especially useful if considering a flying base station to provide emergency networking or futuregeneration networking with greater device/rate requirements for connectivity services) $[10,12,13]$.

Building Security on the Base Station and Networking Edge. Security for $5 \mathrm{G}$ networking can be implemented at multiple levels. The base station or an edge server closer to the attacker user than the core network can build intelligence based on networking/sensed data to detect the injection threats to inform the attack. If such intelligence is real-time (i.e., occurring while the attack is ongoing), the base station can filter the attack traffic for mitigation so that the attack impact does not reach beyond the edge of the network. The base station on the networking edge can also build prioritization of the users, for example, 
based on token credentials from the previous session, so that the priority users demonstrating greater trustworthiness than others can still access the networking service (i.e., not being subjected to DoS). These approaches will mitigate the attack impact, thus reducing the security risk, and remain as a critical research direction to secure mobile/wireless networking.

5G Implementations. Our work studies the security based on the 5G NR standard and discover and exploit the vulnerability from the security being absent initially during the channel access setup before the authentication. A more systems approach based on the $5 \mathrm{G}$ system implementations and the collaborations with a real-world cellular service provider will facilitate more concrete analyses on the attack impact of the injection analyses. Such an approach will also enable the practicality analyses of the future security solutions for $5 \mathrm{G}$ networking.

Securing 6G and Next-Generation Networking. As observed from the mobile networking technology evolutions from $2 \mathrm{G}$ to $5 \mathrm{G}$, mobile networking learn from its past generation of technologies to build the future-generation of networking technologies. Our research in securing the existing mobile networking technologies will contribute to building security in the next-generation technologies, such as $6 \mathrm{G}$. Such research will enable the design of the security mechanisms along with the design of the $6 \mathrm{G}$ technology functionalities so that we can practice the security-by-design principle that would improve the security mechanisms' effectiveness and practicality beyond building security around the existing technologies. It will also drive and enable the security solution incorporation into the standards for wider deployment of the security implementations.

\section{Conclusion}

There is no security protection at the initial parts of the $5 \mathrm{G}$ control communications before the authentication and the security setup. The attacker can exploit this vulnerability to perform injection attack to consume the networking resources of the $5 \mathrm{G}$ service provider infrastructure. In this work, we study the $5 \mathrm{G}$ networking standardization and analyze the injection threat impact against the networking availability. We conduct networking measurements on real-world 5G and estimate the injection threat impacts in communications, hops, and latencies based on the networking measurements. We intend to inform the $5 \mathrm{G}$ standard technologies of the injection threat to encourage and facilitate the R\&D in securing the networking availability. To that end, we include discussions for future work for greater security awareness and potential countermeasures, including the security mechanisms implemented at the base station or the networking edge.

Acknowledgement. This work was supported in part by Institute of Information \& communications Technology Planning \& Evaluation (IITP) grant funded by the Korea government (MSIT) (No.2021-0-00796, Research on Foundational Technologies for $6 \mathrm{G}$ Autonomous Security-by-Design to Guarantee Constant Quality of Security). 
This material is also based upon work supported by the National Science Foundation under Grant No. 1922410.

\section{References}

1. 3GPP. TR 21.915: Release 15 Description; Summary of Rel-15 Work Items (2019). https://www.3gpp.org/release-15

2. 3GPP. TR 21.915: 5G; Procedures for the 5G System (2021). https://www.3gpp. org/specifications/specifications

3. 3GPP. TS 38.211: 5G; NR; Physical channels and modulation (2021). https:// www.3gpp.org/specifications/specifications

4. 3GPP. TS 38.321: NR; Medium Access Control (MAC) protocol specification (2021). https://www.3gpp.org/specifications/specifications

5. Alexa: The top 500 sites on the web (2021). https://www.alexa.com/topsites

6. Arjoune, Y., Faruque, S.: Smart jamming attacks in 5G new radio: a review. In: 2020 10th Annual Computing and Communication Workshop and Conference (CCWC), pp. 1010-1015. IEEE (2020)

7. Bogdanoski, M., Suminoski, T., Risteski, A.: Analysis of the SYN flood dos attack. Int. J. Comput. Netw. Inf. Secur. (IJCNIS) 5(8), 1-11 (2013)

8. Chang, S.Y., Hu, Y.C.: SecureMAC: securing wireless medium access control against insider denial-of-service attacks. IEEE Trans. Mob. Comput. 16(12), 35273540 (2017). https://doi.org/10.1109/TMC.2017.2693990

9. Chang, S.Y., Hu, Y.C., Laurenti, N.: SimpleMAC: a jamming-resilient MAC-layer protocol for wireless channel coordination. In: Proceedings of the 18th Annual International Conference on Mobile Computing and Networking, Mobicom'12, pp. 77-88. Association for Computing Machinery, New York (2012). https://doi.org/ $10.1145 / 2348543.2348556$

10. Chang, S.Y., Kumar, S.L.S., Hu, Y.C., Park, Y.: Power-positive networking: wireless-charging-based networking to protect energy against battery dos attacks. ACM Trans. Sen. Netw. 15(3), 1-25 (2019). https://doi.org/10.1145/3317686

11. Chang, S.Y., Park, Y., Kengalahalli, N.V., Zhou, X.: Query-crafting DoS threats against internet DNS. In: 2020 IEEE Conference on Communications and Network Security (CNS), pp. 1-9 (2020). https://doi.org/10.1109/CNS48642.2020.9162166

12. Desnitsky, V., Rudavin, N., Kotenko, I.: Modeling and evaluation of battery depletion attacks on unmanned aerial vehicles in crisis management systems. In: Kotenko, I., Badica, C., Desnitsky, V., El Baz, D., Ivanovic, M. (eds.) IDC 2019. SCI, vol. 868, pp. 323-332. Springer, Cham (2020). https://doi.org/10.1007/9783-030-32258-8_38

13. Halperin, D., et al.: Pacemakers and implantable cardiac defibrillators: software radio attacks and zero-power defenses. In: 2008 IEEE Symposium on Security and Privacy (SP 2008), pp. 129-142 (2008). https://doi.org/10.1109/SP.2008.31

14. IANA: Internet Assigned Numbers Authority. https://www.iana.org/

15. Kolahi, S.S., Alghalbi, A.A., Alotaibi, A.F., Ahmed, S.S., Lad, D.: Performance comparison of defense mechanisms against TCP SYN flood DDoS attack. In: 2014 6th International Congress on Ultra Modern Telecommunications and Control Systems and Workshops (ICUMT), pp. 143-147. IEEE (2014)

16. Lazos, L., Liu, S., Krunz, M.: Mitigating control-channel jamming attacks in multichannel ad hoc networks. In: Proceedings of the Second ACM Conference on Wireless Network Security, WiSec'09, pp. 169-180. Association for Computing Machinery, New York (2009). https://doi.org/10.1145/1514274.1514299 
17. Lee, P.P., Bu, T., Woo, T.: On the detection of signaling DoS attacks on 3G/WiMax wireless networks. Comput. Netw. 53(15), 2601-2616 (2009)

18. Lichtman, M., Rao, R., Marojevic, V., Reed, J., Jover, R.P.: 5G NR jamming, spoofing, and sniffing: threat assessment and mitigation. In: 2018 IEEE International Conference on Communications Workshops (ICC Workshops), pp. 1-6. IEEE (2018)

19. Negi, R., Rajeswaran, A.: DoS analysis of reservation based MAC protocols. In: IEEE International Conference on Communications, ICC 2005, vol. 5, pp. 36323636 (2005). https://doi.org/10.1109/ICC.2005.1495094

20. Sattar, D., Matrawy, A.: Towards secure slicing: using slice isolation to mitigate DDoS attacks on 5G core network slices. In: 2019 IEEE Conference on Communications and Network Security (CNS), pp. 82-90. IEEE (2019)

21. Silva, R.S., et al.: REPEL: a strategic approach for defending $5 \mathrm{G}$ control plane from DDoS signalling attacks. IEEE Trans. Netw. Serv. Manag. 18(3), 3231-3243 (2020)

22. Sommers, J., Barford, P.: Cell vs. WiFi: on the performance of metro area mobile connections. In: Proceedings of the 2012 Internet Measurement Conference, pp. 301-314 (2012)

23. Tung, Y.C., Han, S., Chen, D., Shin, K.G.: Vulnerability and protection of channel state information in multiuser MIMO networks. In: Proceedings of the 2014 ACM SIGSAC Conference on Computer and Communications Security, CCS'14, pp. 775786. Association for Computing Machinery, New York (2014). https://doi.org/10. 1145/2660267.2660272

24. Yu, C., Chen, S.: On effects of mobility management signalling based DoS attacks against LTE terminals. In: 2019 IEEE 38th International Performance Computing and Communications Conference (IPCCC), pp. 1-8. IEEE (2019)

25. Zhang, T., Lee, R.B.: Host-based DoS attacks and defense in the cloud. In: Proceedings of the Hardware and Architectural Support for Security and Privacy, pp. $1-8(2017)$

Open Access This chapter is licensed under the terms of the Creative Commons Attribution 4.0 International License (http://creativecommons.org/licenses/by/4.0/), which permits use, sharing, adaptation, distribution and reproduction in any medium or format, as long as you give appropriate credit to the original author(s) and the source, provide a link to the Creative Commons license and indicate if changes were made.

The images or other third party material in this chapter are included in the chapter's Creative Commons license, unless indicated otherwise in a credit line to the material. If material is not included in the chapter's Creative Commons license and your intended use is not permitted by statutory regulation or exceeds the permitted use, you will need to obtain permission directly from the copyright holder.

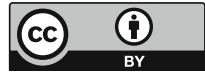

